\title{
Nanomechanical control of an optical antenna
}

\author{
JÖRG MERLEIN ${ }^{\dagger}$, MATTHIAS KAHL ${ }^{\dagger}$, ANNIKA ZUSCHLAG, ALEXANDER SELL, ANDREAS HALM, \\ JOHANNES BONEBERG, PAUL LEIDERER, ALFRED LEITENSTORFER AND RUDOLF BRATSCHITSCH*
}

\author{
Department of Physics and Center for Applied Photonics, University of Konstanz, Universitätsstraße 10, D-78464 Konstanz, Germany \\ tThese authors contributed equally to this work. \\ *e-mail: Rudolf.Bratschitsch@uni-konstanz.de
}

Resonant optical nanoantennas hold great promise for applications in physics and chemistry ${ }^{1-6}$. Their operation relies on their ability to concentrate light on spatial scales much smaller than the wavelength. In this work, we mechanically tune the length and gap between two triangles comprising a single gold bow-tie antenna by precise nanomanipulation with the tip of an atomic force microscope. At the same time, the optical response of the nanostructure is determined by means of dark-field scattering spectroscopy. We find no unique single 'antenna resonance'. Instead, the plasmon mode splits into two dipole resonances for gap sizes on the order of a few tens of nanometres, governed by the full three-dimensional shape of the antenna arms. This result opens the door to new nano-optomechanical devices, where mechanical changes on the nanometre scale control the optical properties of artificial structures.

Metal nanoantennas, the down-scaled optical analogues of radio antennas, are promising systems for applications in nanometre-scale lithography ${ }^{1}$, field-enhanced spectroscopy ${ }^{2}$, nanoscopic light emitters with tailored absorption and emission characteristics ${ }^{3-5}$, light harvesting, high-density optical data storage, and laser-assisted magnetic recording ${ }^{6}$. A second fundamental property of these metallic nanostructures is the extreme sensitivity of the plasmon resonances to their surroundings. Hence, metal nanoantennas may be used in biology and medicine as advanced nanometre-sized biochemical sensors ${ }^{7,8}$. Only recently have optical antenna structures operating in the near-infrared regime of the electromagnetic spectrum been manufactured using electron-beam lithography and focused-ion-beam processing ${ }^{9,10}$. In this work, we present a tunable bow-tie optical nanoantenna fabricated using a colloidal nanomask ${ }^{11-13}$. The structure consists of two gold nanotriangles arranged in a tip-to-tip configuration. The feedgap, the gap between the triangles, can be varied continuously by manipulating one antenna arm with nanometre precision using the tip of an atomic force microscope (AFM), while at the same time the optical response of the nanoantenna is determined with dark-field scattering spectroscopy.

A schematic of the experimental set-up, which enables simultaneous nanomechanical control and nano-optic characterization, is shown in Fig. 1. It consists of two main parts: an AFM for scanning the surface topography and manipulating nanometre-sized objects on a surface, and a dark-field microscope with spectrometer and CCD detector array for recording the scattering spectrum of a single nanosystem.
Figure 2a,b shows the AFM image and dark-field scattering spectrum of one building block of the gold bow-tie antenna, a single equilateral nanotriangle with a side length of $128 \mathrm{~nm}$ and a thickness of $32 \mathrm{~nm}$. For its fabrication using colloidal lithography, the evaporation mask consisted of three touching polystyrene spheres with diameters of $310 \mathrm{~nm}$ on a $170-\mu \mathrm{m}$-thick fused silica substrate. In the AFM image, residues from the fabrication process using the spheres are still visible, but these are no higher than a few nanometres. After evaporation of the gold and removal of the nanospheres, a single metal nanoparticle with a triangular shape remains. We call this specimen a 'nanotriangle' for simplicity. The dark-field scattering spectra for wavelengths from $500 \mathrm{~nm}$ to $1,000 \mathrm{~nm}$ were recorded with excitation and detection polarizations perpendicular to one of the edges of the nanotriangle (Fig. 2b). We found a single resonance in the visible region, which is assigned to the dipole plasmon oscillation in this direction. The resonant behaviour is determined by both the material and the shape of the nanostructure.

The scattering spectrum of the nanotriangle may be calculated using the discrete dipole approximation (DDA). This model enables the simulation of the backscattered spectrum of a threedimensional nano-object by discretizing the particle volume in a series of point dipoles (see Supplementary Information). The dipoles may interact with each other when an external light wave impinges on the structure ${ }^{14}$. We approximate the surroundings of the metal structure assuming a homogeneous medium with a constant index of refraction. Excellent agreement with the experimental scattering spectrum is found if the average values of the indices of refraction of air and fused silica substrate are used in calculations, assuming triangular prisms for the antenna arms (red line in Fig. 2b). Both the resonance position of the plasmon and its width are reproduced quantitatively if the complex dielectric function of gold is taken into account.

In Fig. 2c, the AFM image of a bow-tie nanoantenna consisting of two gold nanotriangles separated by $85 \mathrm{~nm}$ is shown. This structure is generated when four touching nanospheres are used as an evaporation mask. The scattering spectrum for this arrangement is depicted in Fig. 2d, with the polarization of the exciting and detected light set parallel to the long axis of the antenna. As in the case of a single nanotriangle, only one scattering peak is found, at $710 \mathrm{~nm}$. In the following, one antenna arm was moved by an AFM tip so that the total length of the nanoantenna, and hence its feedgap, were gradually 


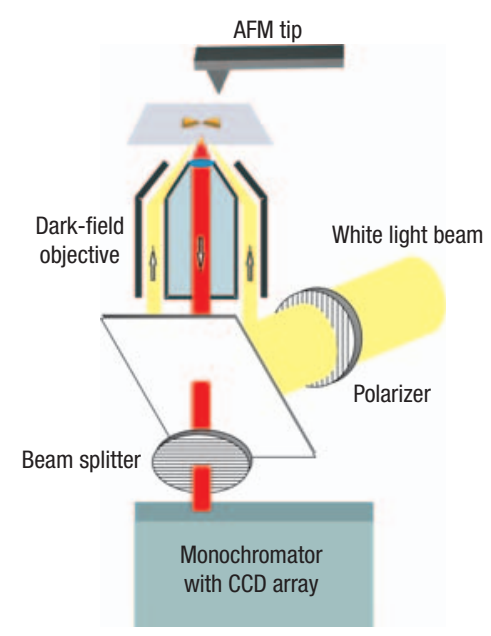

Figure 1 Nano-optomechanical set-up. Schematic drawing of the experimental set-up (not to scale) consisting of an AFM and dark-field scattering spectroscopy system.

reduced. The efficient nanomanipulation of the gold nanotriangle with the AFM tip relies on the fact that gold layers of tens of nanometres thickness only stick to glass substrates feebly ${ }^{15,16}$. After each nanomanipulation step the AFM also recorded an image of the topography of the nanoantenna. The tip radius of the cantilever used was approximately $10 \mathrm{~nm}$. Subsequently, the scanning probe was retracted, and the dark-field scattering spectrum was recorded using free-space optics (Fig. 2e-1). In contrast to all previously reported experiments on nanoantenna structures, our experiment allowed the evolution of the optical properties of the same single nano-object to be studied as a function of position. In our experiment, the feedgap was precisely controlled on a nanometre scale, while the other experimental parameters were kept constant. Most importantly, the shape of the nanoantenna arms was not changed. Reducing the antenna gap to $45 \mathrm{~nm}$ dramatically altered the scattering spectrum (Fig. 2f). The original single plasmon peak split into two distinct resonances. By decreasing the feedgap even further, the resonance at $670 \mathrm{~nm}$ lost strength, and the longerwavelength resonance shifted to the red. This overall trend continued until the tips of the two gold triangles were within a few nanometres of one another. Compared with the starting position (with a gap of $85 \mathrm{~nm}$; Fig. 2d), the long wavelength resonance shifted by $30 \mathrm{~nm}$ when the triangles were almost touching (Fig. $2 \mathrm{j}$ ). In contrast, the short wavelength mode almost disappeared. Finally, when the two antenna arms were pushed into each other (Fig. $2 \mathrm{k}, \mathrm{l}$ ), the main resonance blueshifted to $702 \mathrm{~nm}$. In addition, a long wavelength mode appeared at $940 \mathrm{~nm}$.

The change in the optical properties of a metal nanoantenna with tunable feedgap may be understood in the following way. At a large tip separation of $85 \mathrm{~nm}$, the dark-field scattering spectrum is the superposition of the spectra of the two individual nanotriangles. When the gap decreases, the antenna arms begin to couple electrodynamically. The charge density distribution at any given time in one nanotriangle acts on the other one, and vice versa. As a result, collective plasmon modes form along the entire antenna. The nature and number of plasmon resonances depend on the exact three-dimensional shape of the metal structure. Figure 3 a shows a schematic crosssection of the bow-tie antenna with vertical sidewalls (two triangular prisms) and Fig. $3 \mathrm{~b}$ the same for an antenna with
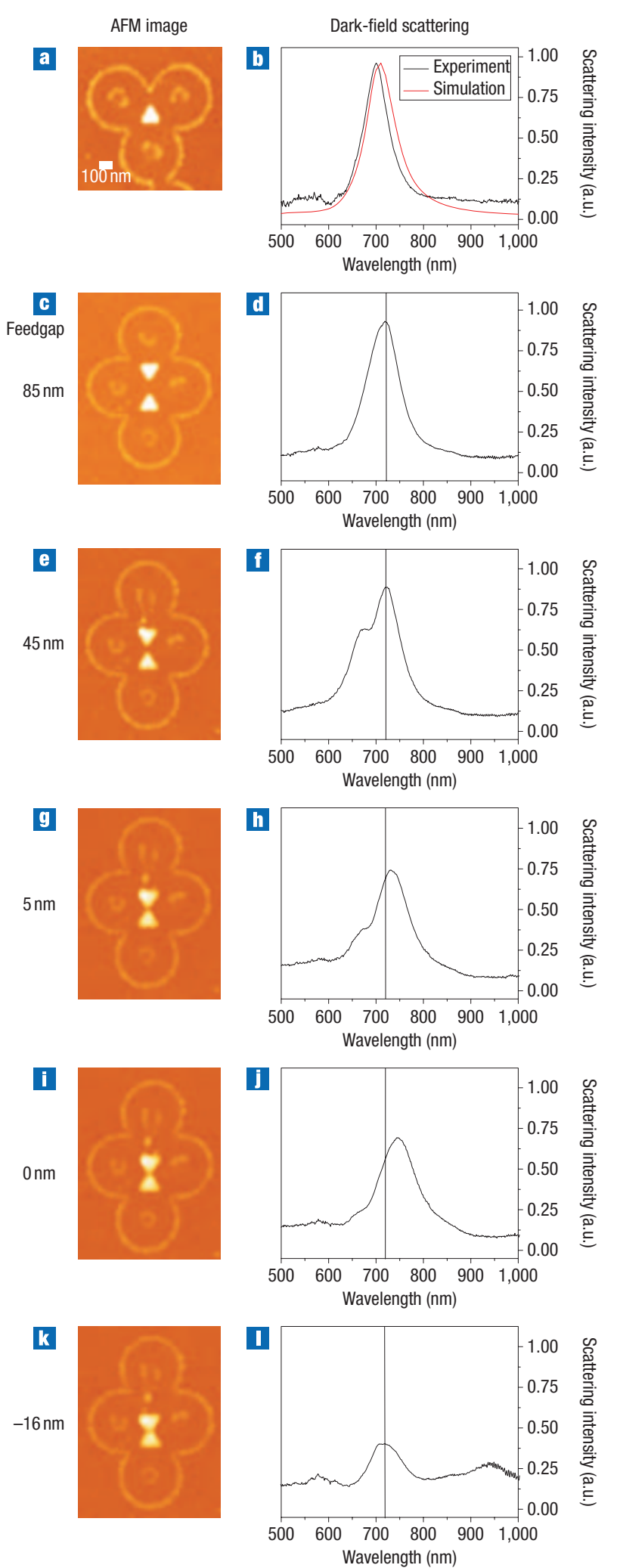

Figure 2 Topography and optical spectroscopy of the tunable nanoantenna. a-I, AFM images (left) and dark-field scattering spectra (right) of a single gold nanotriangle $(\mathbf{a}, \mathbf{b})$ and a single gold bow-tie optical nanoantenna with feedgap sizes ranging from 85 to $-16 \mathrm{~nm}(\mathbf{c}-\mathbf{I})$. In the scattering spectra the optical excitation and detection are polarized along the long antenna axis. The red line in $\mathbf{b}$ is obtained by a DDA calculation (see text for details). 

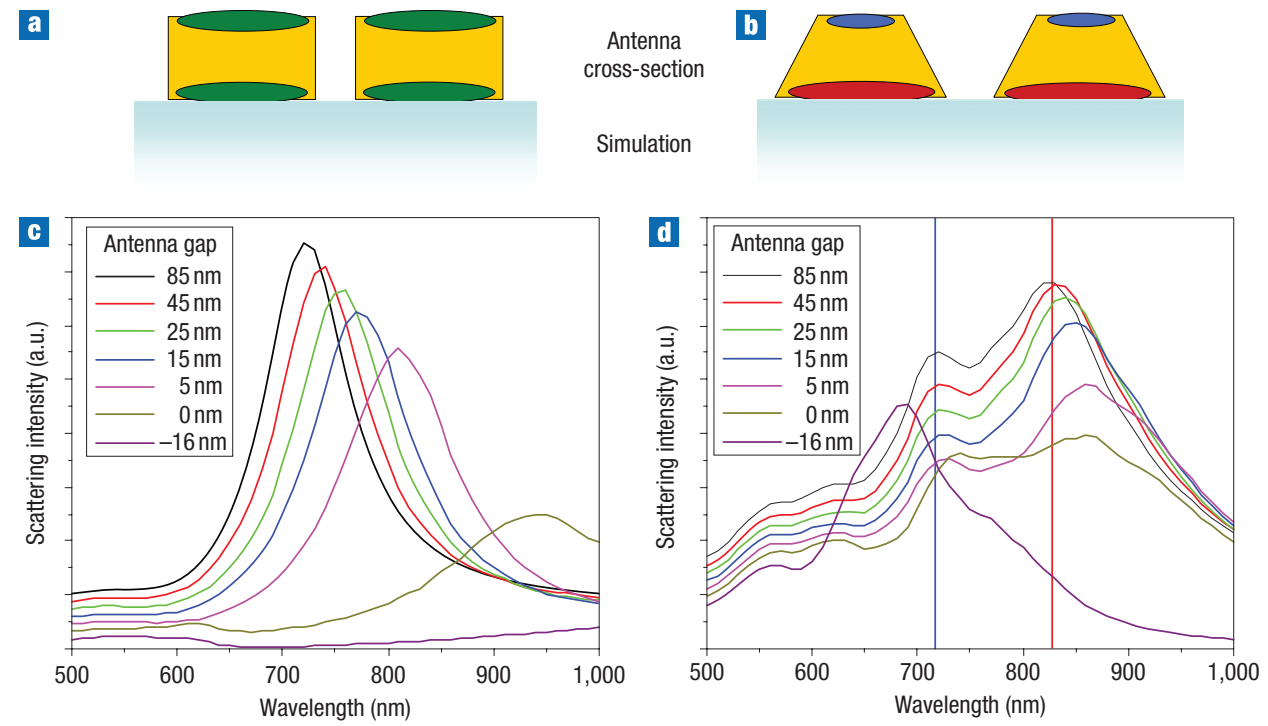

Figure 3 Calculated backscattering spectra depending on the three-dimensional antenna shape. a,b, Schematic drawings of a nanoantenna cross-section for vertical (a) and tilted (b) sidewalls of the antenna arms. The glass/gold surface plasmon is depicted in red, the gold/air surface plasmon in blue. c,d, Calculated backscattering spectra of the gold bow-tie nanoantenna (side length, $128 \mathrm{~nm}$; thickness, $32 \mathrm{~nm}$ ) at different gap sizes assuming vertical sidewalls (c) and tilted sidewalls (dihedral angle base-sidewall, $\left.54^{\circ}\right)(\mathbf{d})$. The excitation and detection polarizations are set in the direction of the long antenna axis.

tilted sidewalls (two truncated tetrahedra). We now calculate the backscattering spectra at different feedgap distances. Antenna arms with vertical sidewalls result in a redshift of a single resonance with smaller gap size (Fig. 3a,c). This behaviour has already been confirmed experimentally for gold nanoantennas that have been fabricated by electron-beam lithography ${ }^{17}$. Tilted sidewalls in the nanotriangles (Fig. 3b,d) break the antenna symmetry perpendicular to the substrate plane (nanotriangle thickness), which causes a splitting of the dipole mode into two localized surface plasmon resonances: one located at the glass/gold interface and the other at the gold/air interface ${ }^{18}$. The different degree of confinement of the localized surface plasmons results in the observed behaviour for decreasing antenna feedgap. The long wavelength mode belonging to the glass/gold plasmon (red vertical line in Fig. 3d) shifts to longer wavelengths with smaller gap sizes, just as in the case of a nanoantenna with vertical sidewalls. The short wavelength mode associated with the stronger confined gold/air surface plasmon (blue vertical line) shows almost no redshift. At smaller feedgaps the scattering cross-section is reduced, because the gold/air surface plasmons in the two nanotriangles are too far apart to couple efficiently (see Fig. 3b). Calculations for the nanoantenna with vertical sidewalls (Fig. 3c) result in a smooth transition from non-touching to touching triangles in the scattering spectra. Eventually, at a triangle separation of $-16 \mathrm{~nm}$, the single dipole resonance has redshifted to $1.34 \mu \mathrm{m}$. In contrast, the tilted sidewall geometry results in a blueshift of the main resonance (Fig. 3d). In the experiment, both features are observed. Of course, the antenna arms pushed into each other are no longer of the idealized shapes assumed in the DDA calculations. We would like to stress that our DDA calculations cannot account for all features in the observed scattering spectra. This finding is most probably due to the assumption of a homogeneous index of refraction for the nanoantenna surroundings in the calculation, whereas in the experiment there is a glass and air half-space, respectively. These shortcomings affect in particular the calculated wavelength positions and the amount of line splitting at different feedgaps. The differences between our model and the experimental situation also become important at tip-to-tip distances of only a few nanometres, where small variations of the tips in the feedgap region strongly modify the scattering spectrum.

With our theoretical model we have gained a qualitative understanding of the plasmon coupling effect for feedgaps in the nanometre regime. In contrast to earlier work on nanoantennas, the full three-dimensional shape has to be taken into account to understand their optical properties. This finding will be particularly important if these antenna structures are resonantly coupled to single nanoemitters, such as semiconductor quantum dots $^{3}$, diamond nanocrystals or single molecules. More sophisticated calculations are needed to obtain quantitative agreement with the experimental data. For example, bulk dielectric functions are used in our simulation. However, as we have seen before, surface effects play a prominent role. Owing to the broken symmetry at the antenna surface, not only the frequency dispersion of the dielectric function $\varepsilon(\omega)$, but also the spatial dispersion $\varepsilon(\omega, k)$ has to be taken into account ${ }^{19-21}$. A theoretical treatment of this effect is beyond the scope of the current work. Nevertheless, the high precision reached in our experiment suggests that spatial dispersion may play a role for the design of plasmonic nanodevices in the near future.

In summary, we have presented a nano-optomechanical study of a gold bow-tie nanoantenna with a variable feedgap. We tune the gap by nanomanipulation with an AFM tip and measure the optical resonances with dark-field scattering spectroscopy. The optical response of the nanoantenna shows two dipole plasmon resonances when the gap sizes are in the regime of a few tens of nanometres. We are able to reliably analyse small changes in the optical properties because, in contrast to previous studies on a series of different nano-objects, we can vary one parameter while keeping the remaining geometry exactly unchanged at the atomic level. The results indicate that the full three-dimensional shape of the nanoantenna has to be taken into account to explain the splittings of the plasmon resonances. This picture is confirmed by DDA calculations with triangular-prism and truncatedtetrahedron shapes for the antenna arms. With our experimental 
technique, the evolution of the optical properties of the same single nano-object as a function of position may be studied. This work has shown, for the first time, how deliberate mechanical changes on the scale of a few nanometres allow control over the optical properties of a nanodevice.

\section{METHODS}

A Zeiss $50 \times$ dark-field objective with a numerical aperture of 0.55 was used. The exciting light from a halogen lamp and the scattered light from the nanoantenna could be linearly polarized and analysed in two orthogonal polarization directions. The scattered light was spectrally resolved by a monochromator with a 150-line grating and a CCD camera. For each scattering spectrum a background spectrum close to the investigated metal antenna was recorded as a reference and subtracted. All spectra were then normalized to the spectral characteristics of the white light source.

Received 25 September 2007; accepted 18 January 2008; published 2 March 2008.

\section{References}

1. Sundaramurthy, A. et al. Toward nanometer-scale optical photolithography: Utilizing the near-field of bowtie optical nanoantennas. Nano Lett. 6, 355-360 (2006).

2. Aizpurua, J. et al. Optical properties of coupled metallic nanorods for field-enhanced spectroscopy Phys. Rev. B 71, 235420 (2005).

3. Farahani, J. N., Pohl, D. W., Eisler, H. J. \& Hecht, B. Single quantum dot coupled to a scanning optical antenna: A tunable superemitter. Phys. Rev. Lett. 95, 17402 (2005).

4. Cubukcu, E., Kort, E. A., Crozier, K. B. \& Capasso, F. Plasmonic laser antenna. Appl. Phys. Lett. 89, 93120 (2006).

5. Novotny, L. Effective wavelength scaling for optical antennas. Phys. Rev. Lett. 98, 266802 (2007).

6. Challenger, W. A. et al. Light delivery techniques for heat-assisted magnetic recording. Jpn J. Appl. Phys. 42, 981-988 (2003).

7. Xu, H. \& Käll, M. Surface-plasmon-enhanced optical forces in silver nanoaggregates. Phys. Rev. Lett. 89, 246802 (2002).
8. Haes, A. J. \& van Duyne, R. P. A nanoscale optical biosensor: Sensitivity and selectivity of an approach based on the localized surface plasmon resonance spectroscopy of triangular silver nanoparticles. J. Am. Chem. Soc. 124, 10596-10604 (2002).

9. Schuck, P. J., Fromm, D. P., Sundaramurthy, A., Kino, G. S. \& Moerner, W. E. Improving the mismatch between light and nanoscale objects with gold bowtie nanoantennas. Phys. Rev. Lett. 94 17402 (2005).

10. Mühlschlegel, P., Eisler, H.-J., Martin, O. J. F., Hecht, B. \& Pohl, D. W. Resonant optical antennas. Science 308, 1607-1609 (2005).

11. Fischer, U. C. \& Zingsheim, H. P. Submicroscopic pattern replication with visible light. J. Vac. Sci. Technol. 19, 881-885 (1981).

12. Deckman, H. W. \& Dunsmuir, J. H. Natural lithography. Appl. Phys. Lett. 41, 377-379 (1982).

13. Burmeister, F. et al. From mesoscopic to nanoscopic surface structures: Lithography with colloid monolayers. Adv. Mater. 10, 495-497 (1998).

14. Draine, B. T. \& Flatau, P. J. User Guide for the Discrete Dipole Approximation Code DDSCAT 6.1. $<$ http://arxiv.org/abs/astro-ph/0409262> (2004)

15. Glang, R. Materials and processes for passive thin-film components. J. Vac. Sci Technol. 3, 37-48 (1966).

16. Haq, K. E., Behrndt, K. H. \& Kobin, I. Adhesion mechanism of gold-underlayer film combinations to oxide substrates. J. Vac. Sci Technol. 6, 148-152 (1969).

17. Sundaramurthy, A. et al. Field enhancement and gap-dependent resonance in a system of two opposing tip-to-tip Au nanotriangles. Phys. Rev. B 72, 165409 (2005).

18. Kelly, K. L., Coronado, E., Zhao, L. L. \& Schatz, G. C. The optical properties of metal nanoparticles: The influence of size, shape, and dielectric environment. J. Phys. Chem. B 107, 668-677 (2003).

19. Landau, L. D. \& Lifshitz, E. M. Electrodynamics of Continuous Media (New York, Pergamon, 1960).

20. Göger, G. et al. Ultrafast spectroscopy of large-momentum excitons in GaAs. Phys. Rev. Lett. 84, $5812-5815$ (2000).

21. Betz, M. et al. Nonlinear optical response of highly energetic excitons in GaAs: Microscopic electrodynamics at semiconductor interfaces. Phys. Rev. B 65, 85314 (2002).

\section{Acknowledgements}

We gratefully acknowledge financial support from the Deutsche Forschungsgemeinschaft (DFG) through SFB 513, the Kompetenznetz Funktionelle Nanostrukturen Baden-Württemberg, and a grant from the Ministry of Science, Research and Arts Baden-Württemberg. We acknowledge the generous help of

M. Fuchs, S. Gerlach, K. Diederichs, R. Stadelhofer and W. Benger by providing extensive computer power for the DDA calculations. We acknowledge the help of H. Ballot and A. Habenicht with the colloidal masks.

Correspondence and requests for materials should be addressed to R.B.

Supplementary information accompanies this paper on www.nature.com/naturephotonics.

Reprints and permission information is available online at http://npg.nature.com/reprintsandpermissions/ 